\title{
ALIMENTACION Y CRECIMIENTO DE JUVENILES DE CORVINA. MICROPOGON OPERCULARIS, EN CONDICIONES EXPERIMENTALES *
}

\author{
Instituto Nacional de Investigacion y Desarrollo Pesquero \\ Mar del Plata, Argentina
}

\section{SYNOPSIS}

The present Daper deals with the results obtained on the basis of studies on the feeding and growth of juveniles of the croaker, Micropogon opercularis in experimental conditions. The following, more important results, were obtained: 1. - The values of the daily food requirement for the juveniles of $79.126 \mathrm{~mm}$ in total length and of $4.1 \cdot 22,3 \mathrm{~g}$ in weight, at the beginning of the experiments, oscillated between 1,29 and $6,88 \%$, with the average of $3,58 \% .2$. - The efficiency of conversion of food is rather high and was calculated; for "normal" specimens as $19,45 \%$ in average. 3. - Very big individual differences were observed in the growth of the juveniles. In the case of the total lengths, the rate of annual growth oscillated between 15,2 and $114,2 \%$ and. in the case of the weights, between 23,5 and $1114.5 \%$. 4. - It was observed that in the scales of the juveniles under experiments the first annulus is not formed or is formed not always in a regular way.
\end{abstract}

\section{Introduccion}

Una de las especies de la familia Sciaenidae de mavor importancia para la pesca en la Argentina, es la corvina blanca o rubia Micropogon opercularis (Quoy y Gaimard, 1824). Se asemeja mucho a la corvina de Brasil Micropogon furnieri (Desmarest, 1822) y en realidad no se sabe con certeza si se trata de dos especies o de una, diferenciada en cierta medida biológicamente en razón de su distribución geográfica, que es bastante amplia. Esta involucra pronunciadas diferencias en lo que se refiere a los factores ambientales. Roux (1973) reemplaza las dos especies por una sola y crea otro género, (Micropogonias furnieri) (Desmarest, 1823), Roux, 1973. Sin entrar en la discusión de sistemática, por razones de necesidad, seguimos com la definición aceptada anteriormente de $M$. opercularis.

La biología de $M$. furnieri de Brasil es bastante bien conocida y existen varios trabajos al respecto, pero en lo referente a corvina en aguas argentinas y uruguayas. M.opercularis, se sabe poco y hay todavia varias lagunas en el conocimiento biológico de esta especie.

En el presente trabajo se analizán los resultados obtenidos del estudio sobre la alimentación y el crecimiento de los juveniles de la corvina en acuarios en determinadas condiciones ambientales. En este estudio se contemplan, entre otros, aspectos tales como la ración diaria del alimento ingerido, o requerimiento alimentario diario, y la eficiencia de conversión del mismo. Estos datos, aportan una valiosa información para el conocimiento de la dinámica de la producción del mar. Por otro lado, teniendo en cuenta las dificultades que se presentan en general a los lectores de la edad de los peces, especialmente en la determinación del primer anillo anual del individuo y de la talla en esta época de vida, se ha pensado que el análisis más detallado del crecimiento del pez durante, por lo menos, el primer año de su vida puede aportar datos de interés para estos estudios.

\section{Material y Metodo}

Los presentes estudios se basaron sobre juveniles de corvina en su prmer año đe vida, capturados en aguas costeras de Mar del Plata, en la pesca comercial de camarón o bien, en una pesca realizada especialmente para tal fin. En su mayoría, el material provino de marzo del año 1977. Estos juveniles, sometidos posteriormente a la cría experimental en acuarios, medían entre 79 y 125 $\mathrm{mm}$ en largo total y entre 62 y $105 \mathrm{~mm}$ em largo standard. El rango del peso oscilaba entre 4,1 y $22,3 \mathrm{~g}$.

Los peces fueron criados todos en condiciones similares, contando cada uno de ellos con un acuario de $60 \times 20 \times 35 \mathrm{~cm}$, provisto de un termómetro $\mathrm{y}$ un burbujeador de aire. Los juveniles fueron mantenidos por separado com el fin de tener un estricto control de la cantidad de alimento ingerido y de evitar cualquer tipo de agresión que se podría producir, hecho muy frecuente, p. ej. en la pescadilla juvenil que se encuentre en captividad. Cada cuatro o cinco días se renovaba el agua de mar, previamente filtrada y acondicionada para tener la misma temperatura que la de los acuarios. Como alimento, en todos los experimentos se utilizó la carne de camarón, tomando en cuenta el hecho de que este crustáceo entra en el régimen alimentario de la joven corvina, de que la cantidad ingerida es fácil de controlar y de que se lo puede obtener sin mayores problemas durante todo el año.
A los peces se los alimentaba todos los días, of reciendo comida en cantidades necessarias para que estos se encuentren saciados y no acepten más alimento. Cada mes, los peces fueron medidos (largo total y largo standard) y pesados en una balanza de presición. La temperatura fué observada todos los días. Debido a las dificultades técnicas, no se pudo mantener una temperatura constante $y$, por lo tanto, aunque se contaba con cierta posibilidad de su regulación, el rango de sus oscilaciones a lo largo de los experimentos, fué bastante amplio. Sin embargo, este hecho fué un tanto positivo que reflejó en cierta medida las condiciones térmicas del mar, que cambian a lo largo del año.

Los experimentos duraron, en ocho casos, más de un año, lo que permitió obtener una cierta información sobre la ración diaria del alimento ingerido y sobre el crecimiento a lo largo de todo ese lapso.

\section{Resultados y Discusion}

Ración alimentaria diaria - La ración alimentaria diaria se calculó como porcentaje del alimento ingerido en un día, relativo al peso total del pez. El valor del mismo se obtuvo como promedio para cada mes. Estos valores oscilaron entre 1,29 y $6,88 \%$. En un sólo caso se comprobó que el juvenil había ingerido, como promedio para un día, el $7,7 \%$ de su peso. Hay que mencionar que esto tuvo lugar cuando la temperatura del agua fué elevada, representando un promedio para el mes de $20,5^{\circ} \mathrm{C},{ }^{\prime}$ con el rango de $19,5^{\circ}-23,0^{\circ} \mathrm{C}$. La gran mayoría de los valores de la ración alimentaria diaria caen entre 3,0 y $4,5 \%$, con el promedio calculado para 60 casos.

Discriminando los datos obtenidos al respecto y tomando en cuenta el tamaño de los peces, se obtienen valores de: $2,55-7,74 \%$ para los juveniles de $4-10 \mathrm{~g}$ de peso; de $1,40-5,83 \%$ para los de $10-30 \mathrm{~g}$ y de $1,67-4.76 \%$ para los de $30-63 \mathrm{~g}$. Como se puede ver, el requerimiento alimentario diario disminuye a medida que el individuo crece.

Los valores hallados en forma experimental, coinciden en cierta medida con los observados en la naturaleza. En los juveniles de la corvina, capturados directamente en el mar, se ha comprobado que el índice de repleción estomacal, (es decir, el peso del contenido estomacal expresado como porciento del peso total de pez), varía entre 0,20 y $7,79 \%$, siendo los más frecuentes los valores de alrededor del $3 \%$ (trabajo en colaboración). Como es lógico, el índice de repleción estomacal, debido a los procesos de digestión, debe ser un poco más bajo que cuando se trata del alimento recién ingerido.

Los datos obtenidos para los juveniles de la corvina son bastante similares a los relativos a los juveniles de la pescadilla, (ynoscion striatus, de la misma zona (Ciechomski \& Ehrlich, 1977). Para los juveniles de este sciénido, se obtuvieron los siguientes valores: En los juveniles de 10,0-22,0 g en las condiciones experimentales, la ración alimentaria diaria fué en promedio de $4,13 \%$. En las condiciones naturales el índice de repleción para los indi viduos de 0,4 a $9,9 \mathrm{~g}$ fué de $1,91 \%$ en promedio, con el rango de $0,12-9,25 \% \mathrm{~g}$ y para los de 10,0 a $25,0 \mathrm{~g}$ fué de $1,36 \%$, con el rango de 0,06 a $5,01 \%$.

\footnotetext{
* Contribución del Instituto Nacional de Investigación y Desarrollo
} Pesquero No 359 . 
Factor de conversion del atimento - Por el factor de conversión del alimento se entiende un índice que determina el porcentaje del peso del alimento que se convierte en el peso del pez.

En la Tabla I están indicados, para 8 corvinas mantenidas durante 1 año, los valores del factor de conversión del alimento en porciento (media anual) y de la ración diaria del alimento ingerido, también expresado como media anual. Podemos advertir una gran diferencia entre todos los individuos, especialmente en lo que se refiere al factor de conversión del alimento. En los casos de los juveniles (b) y (d), estos índices son sumamente bajos, del orden de 4,64 y $4,85 \%$ y en el juvenil (g) de $20,65 \%$, su valor es bastante alto. Como se trata de tas medias anuales, la imagen puede ser, en cierta medida, distorcionada y no siempre refleja exactamente la realidad, en especial cuando se trata de los valores de la ración diaria. De esta manera, no se toman en cuenta los cambios temporarios en el estado fisiológico del pēz que pueden reflejarse an la característica de su alimentación.

Al tratar de establecer una media para el factor de conversión del alimento para todos los juveniles de corvina, dentro del rango del tamaño estudiado, obtenemos el valor de $11.80 \%$. Si desechamos en nuestra media casos tan especiales, aparentemente anormales, de los individuos (b) y (d), el mismo es de $14,15 \%$. Por

TABLA I

TASAS ANUALES DE CRECIMIENTO (en\%) Y CANTIDAD DEL ALIMENTO INGERIDO POR LOS JUVENILES DE CORVINA

\begin{tabular}{|c|c|c|c|c|c|c|}
\hline (1) & (2) & (3) & (4) & (5) & (6) & (7) \\
\hline COR VINA & $\begin{array}{l}\text { Largo total } \\
\text { inicial }(\mathrm{mm})\end{array}$ & $\begin{array}{l}\text { Tasa de crecimiento } \\
\text { anual en largo }(\%)\end{array}$ & $\begin{array}{l}\text { Peso total } \\
\text { inicial }(\mathrm{g})\end{array}$ & $\begin{array}{l}\text { Tasa de crecimiento } \\
\text { anual en peso }(\%)\end{array}$ & $\begin{array}{l}\text { Ración alim. } \\
\text { diaria, media } \\
\text { anual }(\%) \\
\end{array}$ & $\begin{array}{c}\text { Factor de conversión } \\
\text { del alim., medio } \\
\text { anual }(\%)\end{array}$ \\
\hline a & 126 & 49,2 & 22,3 & 240,0 & 2,59 & 11,68 \\
\hline b & 112 & 17,8 & 11,9 & 23,5 & 3,37 & 4,64 \\
\hline c & 109 & 52,8 & 12,8 & 338,2 & 3,81 & 11,77 \\
\hline d & 105 & 15,2 & 11,0 & 44,5 & 3,42 & 4,85 \\
\hline $\mathrm{e}$ & 102 & 94,1 & 9,6 & 587,5 & 4,99 & 15,98 \\
\hline f & 90 & 86,6 & 7,2 & 516,6 & 3,97 & 13,33 \\
\hline $\mathrm{g}$ & 84 & 114,2 & 6,0 & 1114,5 & 3,21 & 20,65 \\
\hline $\mathrm{h}$ & 79 & 79,7 & 4,1 & 700,0 & 5,23 & 11,54 \\
\hline
\end{tabular}

otro lado, al tomar en cuenta lapsos más cortos, de un mes y considerando solamente individuos que se comportaban "normalmente" en el aspecto alimentario, es decir, comiam en forma regular y aumentaban periodicamente en largo y en peso, el valor promedio para 21 casos es de $19,45 \%$, con el rango de 7,10 a $33,3 \%$. Los valores, tanto de $14,15 \%$ como de $19,45 \%$, que parecen estar más cerca de la realidad, especialmente en las condiciones naturales, son más bien altas, al tener en cuenta el clásico valor de $10 \%$, establecido para la transferencia de energía de un nivel al otro en la naturaleza. Al comparar estos datos con los obtenidos para los juveniles de la pescadilla, comprobamos que en este sciénido, para los individuos considerados "normales" en el aspecto alimentario, el valor dęl factor de conversión del alimento es de $18,4 \%$ (Ciechomski \& Ehrlich, 1977).

Crécimiento - En la Figura 1 está representado el crecimiento de 8 juveniles de corvina a través del año en largo y , en la F́ig. 2, el crecimiento en peso.

Como se puede observar, al igual que en el caso del factor de conversión del alimento, se notan enormes diferencias entre los individuos (ver Tabla 1). Analizando los valores de las columnas (3) y (5) que indicam la tasa de crecimiento anual en lango y en peso, comprobamos que estos oscilan entre 15,2 y $114,2 \%$ para el largo y entre 23,5 y 1114,5 para el peso. Para los casos extremos, se obtuvo el siguiente cuadro: Un juvenil de corvina de $105 \mathrm{~mm}$ y de $11,0 \mathrm{~g}$, alcanzó, al cabo de un año de experiencia, el largó de $121 \mathrm{~mm}$ y el peso de $15,9 \mathrm{~g}$, mientras que otro, mantenido simultaneamente en acuario durante un año, en las mismas condiciones, que medía $84 \mathrm{~mm}$ y pesaba $6,0 \mathrm{~g}$, alcanzó en el mismo lapso, el largo de $180 \mathrm{~mm}$ y un peso de $74,6 \mathrm{~g}$. Respecto a la relación entre la tasa de crecimiento del pez y la temperatura del agua, no se ha podido establecer referencias bien definidas. Sin embargo, al analizar los gráficos de las Figuras 1 y 2, se observa que el aumento en largo $y$, especialmente, en peso, se hace mayor a partir de setiembre-octubre, cuando sube la temperatura. Este aspecto merece estudios más detallados que requieren más material y más posibilidad de regulación de las temperaturas.

Estas observaciones, ademas del interés biológico, tienen importancia para los que estudian la edad y crecimiento de los peces $y$, en este caso preciso de la corvina. En estos estudios, una de las cosas más importantes $\mathrm{y}$, a veces dificultosas, es la determinación del primer anillo anual en la escama e en el otolito del pez y del largo que tiene el individuo al formar esta marca anual. En nuestro caso, en los individuos que forzosamente tendrían que tener 1 anillo anual, el largo oscila entre 121 y $180 \mathrm{~mm}$. Aunque las condiciones experimentales no reflejan exactamente las naturales, el hecho es significativo y tendría que ser considerado en el estudio del crecimiento y de la edad de los adultos. Según los datos suministrados en el trabajo de Erhardt \& A rena (1977), en el cual los autores consideran, entre otras cosas, el crecimiento de la corvina en el Río de La Plata, los individuos con 1 anillo anual tienen en promedio un largo de alrededor de $18 \mathrm{~cm}$ y el peso cerca de $80 \mathrm{~g}$.

Los anillos anuales en la corvina, tanto en sus escamas como en los otolitos, se formarían con más probabilidad en el invierno. Según Vazzoler (1971) en la corvina Micropogon furnieri de Brasil, los anillos anuales en la población del área subtropical del norte $\left(23^{\circ} \mathrm{S}-29^{\circ} \mathrm{S}\right)$ se forman en el verano $\mathrm{y}$ en la del área templada del sur $\left(29^{\circ} \mathrm{S}-33^{\circ} \mathrm{S}\right)$ se forman entre el fin del otoño e invierno. 


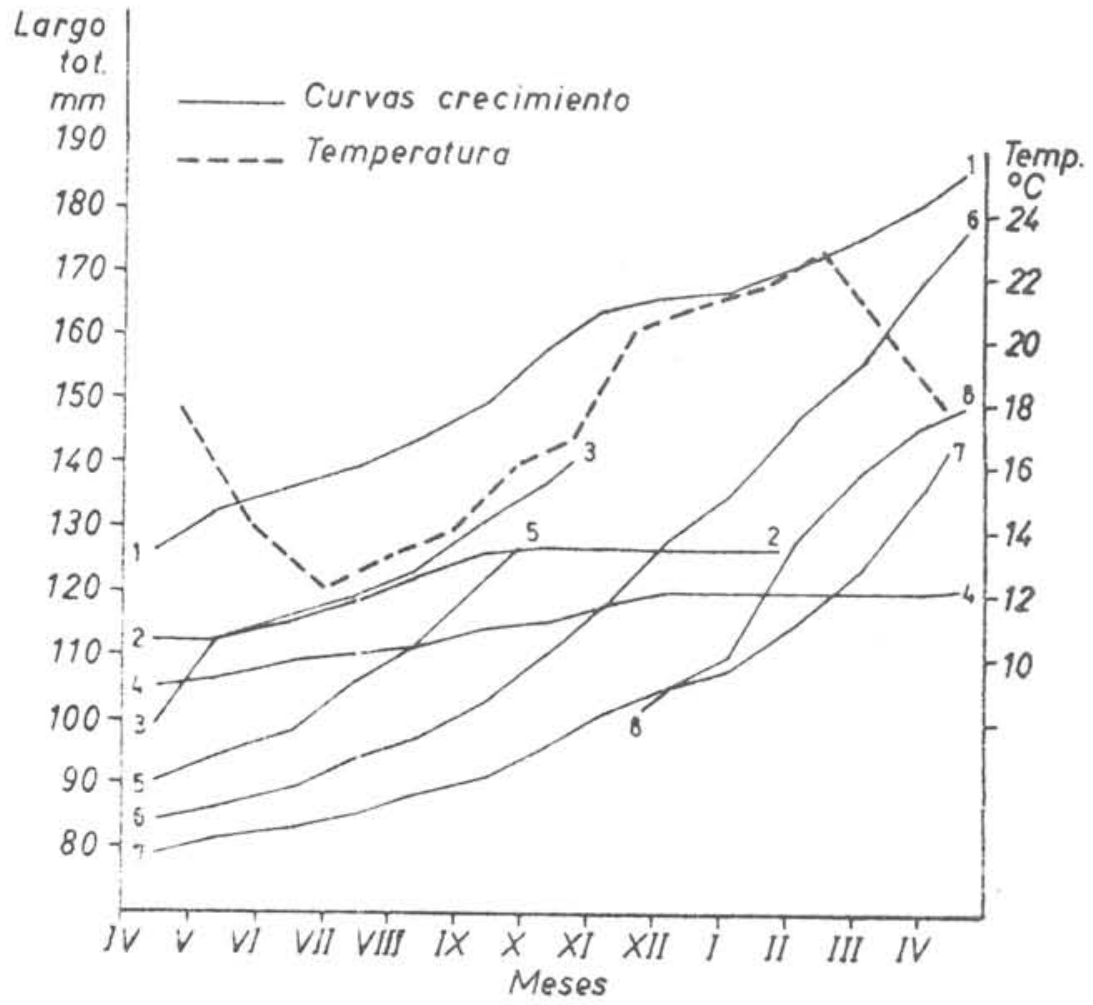

lig C C recimiento en largo total de juveniles de la corvina, a través del año.

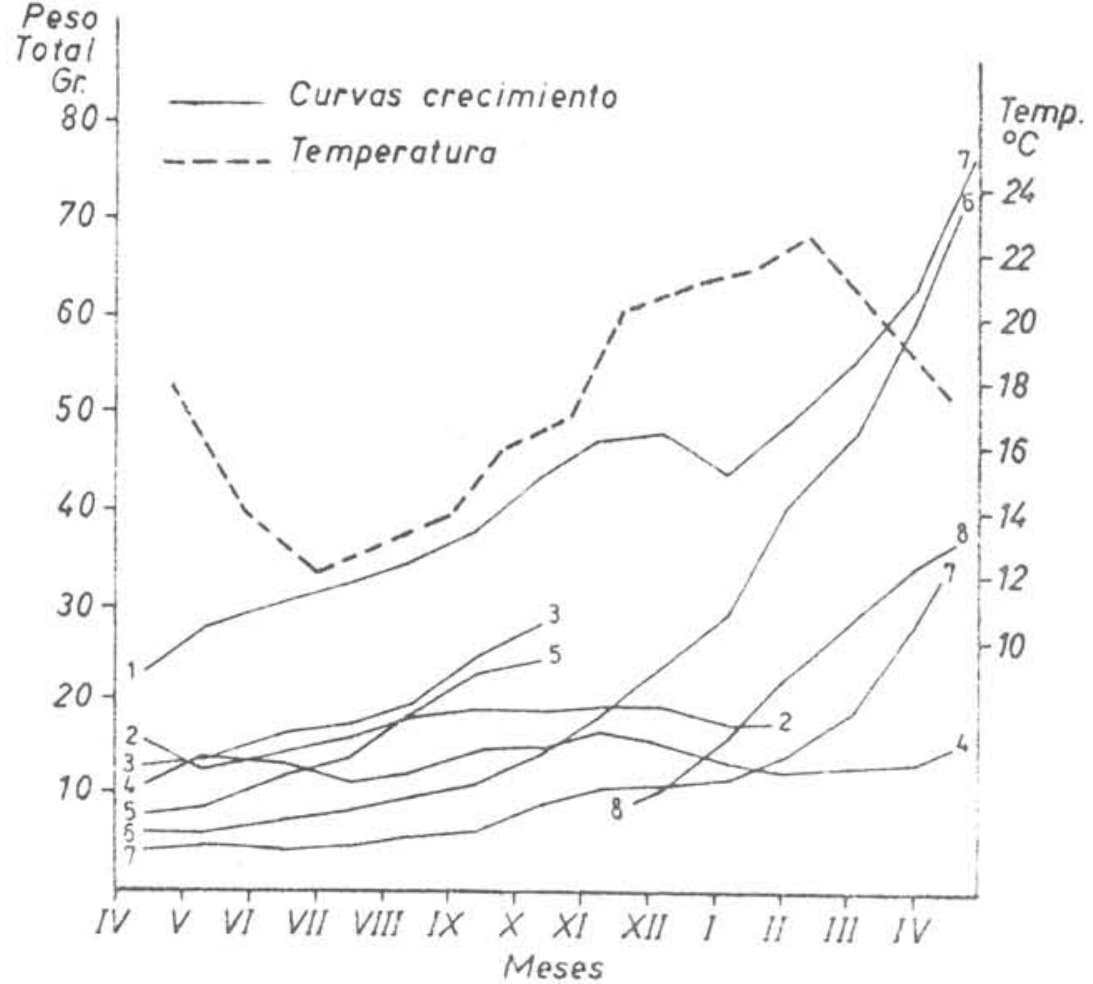


Joseph (1962) ha establecido que en dos especies de sciénidos, Roncador stearnsi y Menticirrhus undulatus en las aguas del Pacífico, los anillos anuales en las escamas se forman al final del invierno y principio de la primavera.

Con el fin de comprobar si en las escamas de nuestras corvinas se han formado los anillos anuales durante más de un año de cautiverio, al fin de las experiencias se examinaron las escamas extraídas a la altura de la aleta dorsal anterior, por encima de la línea lateral. Con este propósito, se han estudiado ocho individuos con la permanencia de, por lo menos, un año los acuarios. El resultado fué el siguiente: en cinco de ellas, que se caracterizaban por el comportamiento "normal" en lo que se refiere a la alimentación, no se ha podido establecer ningún tipo de anillo. La estructura de la escama presentaba una imagen parecida a la de un juvenil pequeño, capturado en el mar, que no ha podido todavía formar su anillo anual. La única diferencia que se observaba residía en los tamaños de las escamas. En otra de las corvinas "normales", se pudo detectar un anillo muy tenue, que correspondió a los meses de julio-agosto, lo que se determinó por retrocálculo. Hay que subrayar, sin embargo, que en estos meses, el juvenil mencionado ha ingerido menos alimento que en los otros meses del año. En dos restantes corvinas, que comieron relativamente poco y aumentaron en forma muy escasa, tanto en largo como en peso, se ha comprobado la existencia de dos anillos tenues, de los cuales uno correspondía al momento de la captura y comienzo del cautiverio (abril). Estas corvinas, durante el lapso señalado, aceptaban el alimento en forma muy limitada.

La dificuldad de determinar los anillos anuales en las escamas de nuestra corvina, fué señalada por Haimovici (1974) quien trató de determinar la edad de los adultos. Sin embargo Vazzoler (1962) y Vazzoler (1971) han utilizado las escamas de Micropogon furnieri para la interpretación de la edad con resultados positivos.

Los datos obtenidos y presentados en este trabajo son todavia escasos y no esclarecen en forma satisfactoria los problemas relativos al crecimiento y la alimentación de la corvina en su primer año de vida. Debido a la complejidad de estos problemas y ra importancia que está involucrada en el conocimiento más profundo de los mismos, se necesitan estudios experimentales más detallados y de mayor envergadura

\section{Referencias Bibliográficas}

CIECHOMSKI, J. de \& EHRLICH, M. D. 1977. Alimentación de los juveniles de pescadilla, Cynoscion striatus (Cuvier, 1829) Jordan \& Evermann, 1889 en el mar y en condiciones experimentales. Pisces. Sciaenidae. Physis, B. Aires, 37(93): 1-12.

ERNARDT, N. M. \& ARENA, G. 1977. Dinámica de población de corvina blanca (Micropogon opercularis) en el Río de La Plata y su frente oceánico. Infme téc., Inst. nac. Pesca, Uruguay, (8): $1-45$.

HAIMOVICI, M. 1974. Resultados preliminares sobre la biología y crecimiento de la corvina rubia (Micropogon opercularis) del área de Mar del Plata. CARPAS/6/74/Tec., (9): 1-12 (mimeo.).

JOSEPH, D. C. 1962. Growth characteristics of two southern California surffishes, the California corbina and spotfin croaker, family Sciaenidae. Fish Bull. Calif., (119): 1-54.

ROUX, C. 1973. Poissons téléostéens du plateau continental brésilién. Resultats scientifiques des campagnes de la " $\mathrm{Ca}$ lypso", 10. Annls Inst. océanogr., Monaco, n. s., 49 (fasc. supplem.): 23-207.

VAZZOLER, A. E. A. de M. 1971. Diversificação fisiológica e morfológica de Micropogon furnieri (Desmarest, 1822) ao su de Cabo Frio, Brasil. Bolm Inst. oceanogr., S Paulo, 20(2): $1-70$.

VAZZOLER, G. 1962. Sôbre a biologia da corvina da costa sul do Brasil. Bolm Inst. oceanogr. S. Paulo, 12 (1): 53-102. 